\title{
On Improving the Classification of Myocardial Ischemia Using Holter ECG Data
}

\author{
MW Zimmerman, RJ Povinelli
}

\author{
Marquette University, Milwaukee, WI, USA
}

\begin{abstract}
In this paper, a method is proposed to improve an algorithm for myocardial ischemia classification created by Langley et al. The Langley classifier achieves a very high sensitivity $(99.0 \%)$, but a lower specificity value (93.3\%). In order to improve the specificity, the proposed algorithm attempts to reclassify the events that the Langley classifier labels ischemic. The classifier used is a support vector machine. The features used are the mean of the ST deviation, maximum value of the ST deviation, and the initial ST deviation.

The classifier is able to increase the specificity from $92.3 \%$ to $93.3 \%$. The drawback is that the sensitivity is reduced from $99.0 \%$ to $97.5 \%$. This causes the overall accuracy to decrease slightly from 95.6 to 94.8 .

The algorithm shows promise in being able to increase specificity, but work must be done to find features that do not cause such a large decrease in the sensitivity.
\end{abstract}

\section{Introduction}

Heart disease is the leading cause of death in the United States. The most common type of heart disease is myocardial ischemia, which is caused by a blockage in the arteries leading to the heart [1]. This type of blockage deprives the cardiac tissue of necessary oxygen. Without oxygen, the cardiac tissue begins to die leading to a myocardial infraction or heart attack.

In order to prevent myocardial infarction, the signs of ischemia must be quickly and accurately detected. Detection of ischemia can be accomplished with coronary angiography, the most accurate, but also the most invasive method used for detection [2]. Exercise testing and echocardiograms are other common detection schemes. Unfortunately, because these methods are expensive and often very invasive, they are generally only performed on high-risk patients.

This means that many patients do not receive testing. If a patient who is not deemed high-risk experiences silent ischemia (condition that does not induce chest pain) the condition will most likely not be detected. If a more affordable, easier to administer testing scheme were available, more patients might have the opportunity to be tested.
In order to develop a method for detection of ischemia that is both accurate and easy, research has focused on the use of electrocardiogram (ECG) recordings [3-5]. The electrocardiogram is a recording of the electrical fronts transversing the tissues of the heart. These electrical fronts trigger the mechanical pumping action of the cardiac muscles [6].

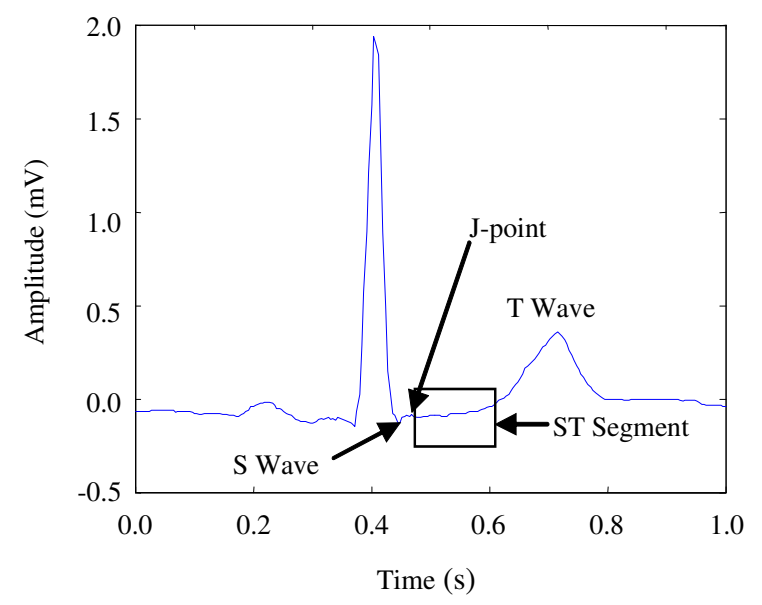

Figure 1 - ECG beat with important points labeled

Research has shown that as the cardiac tissue is deprived of oxygen, changes occur in the electrical signals of the electrocardiogram [7, 8]. This is most evident during ventricular repolarization. The damaged cardiac tissue does not depolarize as quickly as the healthy tissue. This causes some of the depolarizing wave to appear during the normally isoelectric ST segment (the time between the $S$ wave and the $T$ wave). If the damage is severe enough, it may even affect the $\mathrm{T}$ wave. By using features derived from the modified ST segment and T wave it may be possible to determine if a patient is experiencing ischemia $[7,8]$.

Using the information regarding ST segments and ischemia, attempts have been made at developing classification techniques that use ECG signals. One such method, developed by Langley et al, uses threshold levels and time durations to classify ischemic events [5]. The method produced $99.0 \%$ sensitivity when classifying the 'Test Set' of the Long-Term ST Database and had a 
specificity of $92.3 \%$. This algorithm won the 2003 Computers in Cardiology challenge.

This paper uses a support vector machine classifier to reclassify the events that were labeled as ischemia by the Langley method. This classification method will use features from the ST segment and $\mathrm{T}$ wave. The time at which an ST event has occurred will be supplied, so no event detection will be necessary. The goal is to improve the sensitivity of the Langley method.

\section{Long-term ST database}

The Long-Term ST Database consists of 86, two or three lead (signal), 21 to 24 hour, Holter ECG recordings. These recordings are portioned into a 'Training Set' and a 'Test Set'. The publicly available 'Training Set' consists of 43 records from 42 patients. There are 1292 events to be labeled in the 'Training Set.' The 'Test Set' is not publicly available. This set consists of 43 recordings from 38 patients. There are 1974 events to be labeled in the 'Test Set' [9].

Complete annotations have been provided for the database. These annotations label the significant ST shifts and episodes. The beginning (J-point) of most ST segments has been annotated along with $\mathrm{R}$ wave annotations using a 16 second averaging window. These annotations have been generated using only the first channel of each patient's ECG recording. In cases where the first lead is too noisy to allow for detection, a second detection was made using the WQRS application available in the WFDB applications package from PhysioNet [10]. The WQRS program was applied to the signal on which the challenge specified that the episode was seen.

To aid in the development of an ischemia classification algorithm, complete ST level annotations have also been provided. These annotations give the ST level, ST reference function, and the calculated ST deviation. The ST reference is a expertly labeled moving average of the important ST shifts. The ST deviation is calculated by subtracting the ST level from the ST reference function [9]. See Figure 2 for an example of how ST deviation is calculated.

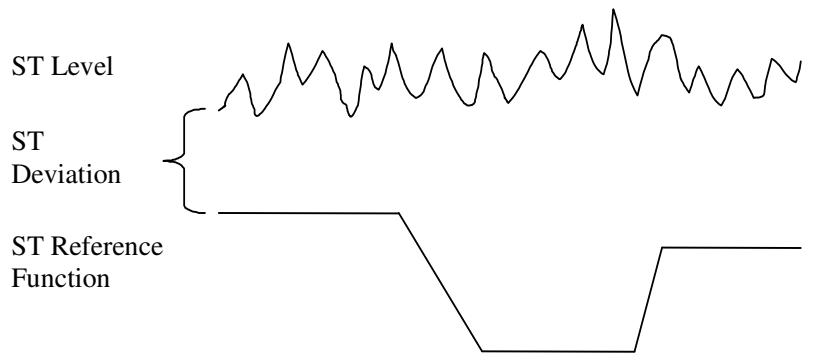

Figure 2 - Example ST deviation calculation

\section{Support vector machine theory}

The support vector machine (SVM) is a method for dividing a feature space using an optimized hyperplane. The goal is to minimize both error and complexity of the classifier. The benefit of this functionality is that SVMs are excellent for modeling spaces with relatively small amounts of data [11].

The simplest implementation of the support vector machine uses two classes $(+1,-1)$. The +1 class is assigned to a positive diagnosis, for example ischemic causes. The -1 is assigned to the negative class, for example non-ischemic causes. If the feature space of these two classes is linearly separable, a hyperplane can be used to divide the data. If, however, the feature space is not linearly separable, the space can be projected into a higher dimensional metric space, known as the Hilbert space through the use of a kernel function. The goal of this transformation is to find a dimension where the feature space will be linearly separable. The formula for the hyperplane that divides the Hilbert space is:

$$
a, \hat{y}=\operatorname{sign}\left(\sum_{i=1}^{N} \alpha_{i} y_{i} K\left(x, x_{i}\right)+b\right)
$$

where $x$ is a vector of inputs, $K\left(x, x_{i}\right)$ is the kernel function, $\alpha_{i}$ is the set of Lagrange multipliers or weights, $b$ is a bias term, $y_{i}$ is the input classes, and $\hat{y}$ is the classification estimate. This equation estimates the hyperplane that generates the largest margin between the two classes [12].

One common kernel function is the radial basis function $(\mathrm{RBF})$. The RBF is defined by:

$$
K\left(x, x_{i}\right)=e^{-\left|x-x_{i}\right|^{2} / 2 \sigma^{2}} .
$$

The parameters for equations (1) and (2) are learned from the training data set. One advantage of the radial basis function is that it is unaffected by shifting of the data. This advantage is very important when applying the support vector machine to ECG data because the waveforms between different patients are often significantly shifted [12].

\section{Langley algorithm}

The Langley classifier uses threshold levels to classify ST events as ischemic or non-ischemic. To be classified as ischemic, an ST event must meet certain criteria before it is determined to be over. First, the ST deviation (difference between deviation and a calculated reference function) must be greater than $50 \mu \mathrm{V}$ at the event start. The deviation must then increase to above $100 \mu \mathrm{V}$ and remain above $100 \mu \mathrm{V}$ for at least 30 seconds. The event is determined to end when the deviation drops below $50 \mu \mathrm{V}$ for at least 30 seconds [5]. Figure 3 shows a graphical representation of how the Langley method functions. 


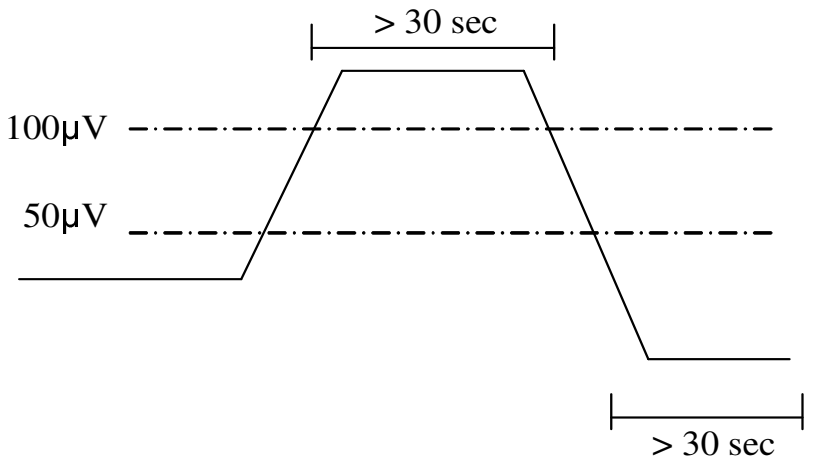

Figure 3 - Example of how Langley method is computed

Axis shifts and conduction changes do not create dynamics that follow the criteria described above. In fact, they are not considered to have a specific end time. For these reasons, the axis shift and conduction change events are accurately classified as non-ischemic. Heart raterelated changes, however, do generate ST deviations that sometimes meet this criteria. When heart rate related shifts are classified as ischemia, they decrease the specificity.

The accuracy results for the Langley et al method are shown in Table 1 . These results were calculated by applying the method to the 'Test Set' of the Long-Term ST Database.

Table 1 - Langley method accuracy results for 'Test Set'

\begin{tabular}{ccc}
\hline Sensitivity & Specificity & Accuracy \\
\hline $99.0 \%$ & $92.3 \%$ & $95.6 \%$ \\
\hline
\end{tabular}

\section{Algorithm}

The first step in implementing the proposed algorithm is to train the support vector machine classifier. The training events are first fed to the Langley classifier. Events classified as non-ischemic are discarded during the training process. The features to be used in classification are then extracted from the events labeled as ischemic.

Based on the researched correlation between ST deviation and ischemia, the features used in the proposed algorithm are derived from the ST segment of the ECG waveform. The features used are:

- Maximum ST deviation

- Mean ST deviation

- Initial ST deviation

Calculation of the features is very straightforward. The max of the ST deviation is the maximum ST deviation between the given start time and the calculated end-time. Likewise, the mean is calculated using the ST deviation during the discovered event time. The initial ST deviation is simply the value of the ST deviation at the given event start time.

Once all the features have been extracted, they are placed into a vector space. A vector containing the expert classifications of the events is also created. These two vectors are fed into the support vector machine for training.

The radial basis function (RBF) is used as the kernel of the support vector machine. The width of the kernel is determined using integer products of the mean of the distance from each of the positive points to its nearest negative point in the training set (nearest neighbor). This heuristic gave a value of approximately 34 for the 'Training Set.' The results sections shows the accuracies for each of the integer multipliers of the kernel width.

Given the features and kernel parameters the support vector machine uses the iterative method described in section 3 to discover the hyperplane that creates the largest margin between the two classes in the higher dimensional space created by the kernel.

The 'Test Set' ST event beats are classified by comparing their ST features with the hyperplane-divided space of the support vector machine. The event is classified based on which side of the hyperplane the point created by the feature-set falls.

\section{Results}

The accuracy results in Table 2 show that the proposed method is able to successfully increase the specificity from $92.3 \%$ (Langley method) to $94.3 \%$ when the kernel width is 34 . One drawback of this approach is that the sensitivity is reduced from $99.0 \%$ to $80.5 \%$ respectively. These values produce an overall accuracy of only $89.3 \%$ compared with $95.6 \%$ percent for the Langley method.

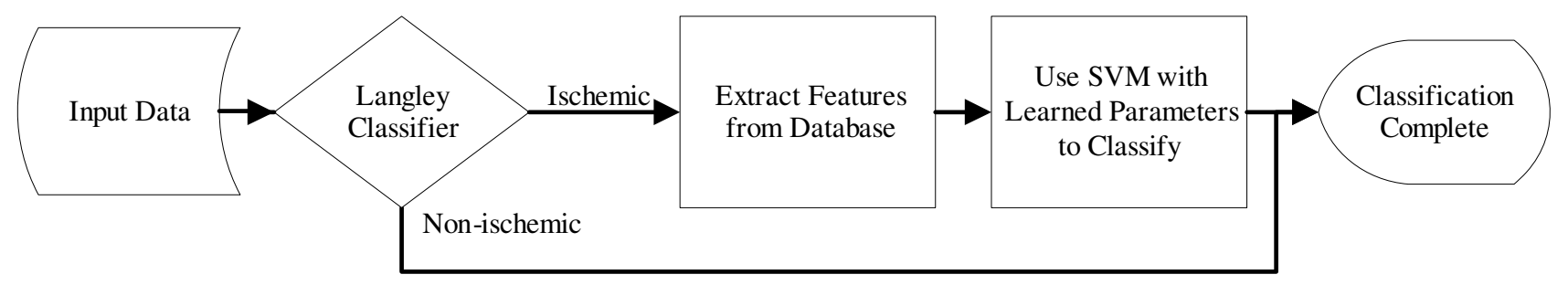

Figure 4 - Flowchart of the proposed algorithm 


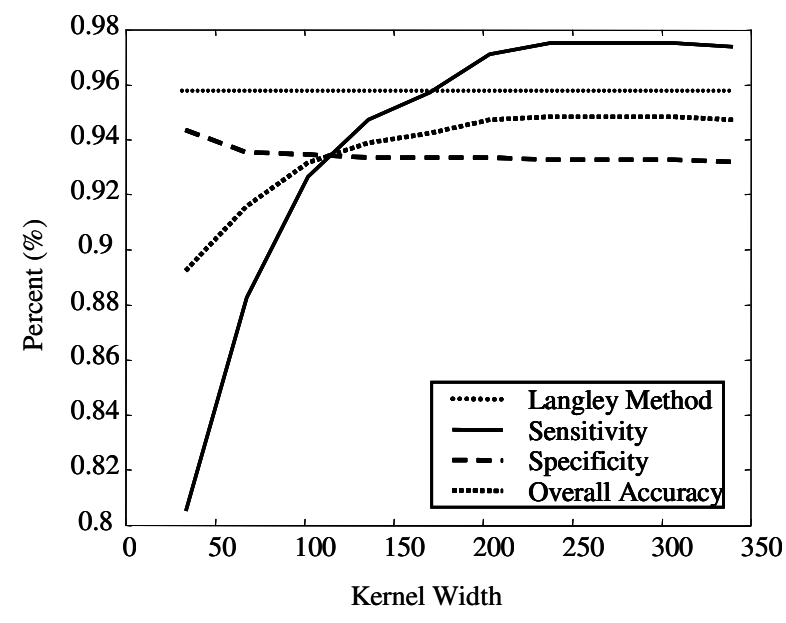

Figure 5 - Results for various kernel widths

The best overall accuracy of $94.8 \%$ occurs when the kernel width is between 238 and 306. The sensitivity of $97.5 \%$, at these widths, is slightly lower than the Langley method, while the specificity of $93.3 \%$ is $1 \%$ higher. Overall, the method was not able to surpass the overall accuracy of the Langley method, which was $95.6 \%$.

Table 2 - Accuracy results for particular values of the kernel width

\begin{tabular}{rrrr}
\hline $\begin{array}{r}\text { Kernel } \\
\text { Width }\end{array}$ & Sensitivity & Specificity & Accuracy \\
\hline 34 & $80.5 \%$ & $94.3 \%$ & $89.3 \%$ \\
68 & $88.2 \%$ & $93.5 \%$ & $91.6 \%$ \\
102 & $92.7 \%$ & $93.4 \%$ & $93.2 \%$ \\
136 & $94.7 \%$ & $93.4 \%$ & $93.9 \%$ \\
170 & $95.7 \%$ & $93.4 \%$ & $94.2 \%$ \\
204 & $97.1 \%$ & $93.4 \%$ & $94.7 \%$ \\
238 & $97.5 \%$ & $93.3 \%$ & $94.8 \%$ \\
272 & $97.5 \%$ & $93.3 \%$ & $94.8 \%$ \\
306 & $97.5 \%$ & $93.3 \%$ & $94.8 \%$ \\
340 & $97.4 \%$ & $93.2 \%$ & $94.7 \%$ \\
\hline
\end{tabular}

\section{Discussion}

The results from the proposed algorithm for myocardial ischemia classification show that the algorithm is not able to improve the method developed by Langley et al. The best overall accuracy is still $0.8 \%$ lower. The algorithm does, however, succeed in increasing the specificity by as much as $2 \%$.

The main problem with this algorithm is that the tradeoff that occurs between specificity and sensitivity is too great. Increasing the specificity causes the sensitivity to drop, decreasing the overall accuracy significantly.

The overall framework for this algorithm does seem to show some promise, as it is able to increase the specificity. It appears, however, that in this case, the features used might be too closely related to those of the Langley classifier. Since the errors made in both classifiers are the same, the accuracies are not increased.

In the future, experimentation should be done to find new features which are completely independent of the ST deviation values.

\section{References}

[1] Preventing heart disease and stroke: Centers for Disease Control and Prevention, 2004.

[2] Baum S, Pentecost MJ. Abram's angiography. Philadelphia: Lippincott-Raven, 1996.

[3] Diamantaras KI, Stamkopoulos T, Maglaveras N, Strintzis M. ST segment nonlinear principal component analysis for ischemia detection, Computers in Cardiology, Indianapolis, IN, 1996;23:493-496.

[4] Silipo R, Marchesi C. Artificial neural networks for automatic ECG analysis. IEEE Transactions on Signal Processing 1998;46:1417-1425.

[5] Langley P, Bowers E, J Wild MD, et al. An algorithm to distinguish ischaemic and non-ischaemic ST changes in the holter ECG, Computers in Cardiology, Thessaloniki, Greece, 2003;30:239-242.

[6] Wagner GS. Marriott's practical electrocardiography. Baltimore, MD: Williams \& Wilkins, 1994.

[7] Braunwald E, Maroko P. ST-segment mapping. Realistic and unrealistic expectations. Circulation 1976;54:529-532.

[8] Janse M, Kleber A. Electrophysiological changes and ventricular arrhythmias in the early phase of regional myocardial ischemia. Circulation Research 1981;49:10691081.

[9] Jager F, Taddei A, Moody G, et al. Long-term ST database: A reference for the development and evaluation of automated ischaemia detectors and for the study of the dynamics of myocardial ischaemia. Medical and Biological Engineering and Computing 2003;41:172-182.

[10] Moody GB. WFDB applications guide. Vol. 2003 Cambridge, MA, 2003.

[11] Sebald DJ, Bucklew JA. Support vector machine techniques for nonlinear equalization. IEEE Transactions on Signal Processing 2000;48:3217-3226.

[12] Herbrich R. Learning kernel classifiers: Theory and algorithms. Cambridge, Mass: MIT Press, 2002.

Address for correspondence.

Richard J. Povinelli

EECE Department, Marquette University

1515 W. Wisconsin Ave.

Milwaukee, WI 53233

richard.povinelli@marquette.edu 